\title{
Social Cognition Deficits Are Pervasive across Both Classical and Overlap Frontotemporal Dementia Syndromes
}

\author{
Faheem Arshad $^{a} \quad$ Avanthi Paplikar $^{a}$ Shailaja Mekalab Feba Varghese ${ }^{a}$ \\ Vandana Valiyaparambath Purushothaman ${ }^{c}$ Darshini Jeevandra Kumar ${ }^{c}$ \\ Leena Shingavi ${ }^{a}$ Seena Vengalila Subasree Ramakrishnan ${ }^{a}$ Ravi Yadava \\ Pramod Kumar Pal ${ }^{a}$ Atchayaram Nalini ${ }^{a}$ Suvarna Alladi ${ }^{a}$ \\ ${ }^{a}$ Department of Neurology, National Institute of Mental Health and Neurosciences, \\ Bangalore, India; ${ }^{b}$ Department of Neurology, Nizam's Institute of Medical Sciences, \\ Hyderabad, India; 'Department of Speech Pathology and Audiology, National Institute of \\ Mental Health and Neurosciences, Bangalore, India
}

\section{Keywords}

Emotion recognition - Empathy · Frontotemporal dementia - Semantic dementia - Social cognition

\begin{abstract}
Objectives: Frontotemporal dementia (FTD) syndromes are a complex group of disorders characterised by profound changes in behaviour and cognition. Many of the observed behavioural abnormalities are now recognised to be due to impaired social cognition. While deficits in emotion recognition and empathy are well-recognised in behavioural-variant (Bv)FTD, limited information exists about the nature of social cognitive impairment in the language variant primary progressive aphasia (PPA) that includes progressive non-fluent aphasia (PNFA) and semantic dementia (SD), and in the motor variants FTD amyotrophic lateral sclerosis (FTD-ALS) and FTD progressive supranuclear palsy (FTD-PSP). This prospective study sought to explore the nature and profile of social cognition deficits across the spectrum of FTD. Methods: Sixty patients on the FTD spectrum, i.e., classical (16 with BvFTD and 20 with PPA) and overlap FTD syndromes (13 with FTD-ALS and 11 with FTD-PSP) were evaluated by means of the social cognition tasks, the Interpersonal Reactivity Index (IRI) for empathy, and pictures of facial affect (POFA) for emotion recognition. General cognition and behaviour were also assessed. Results: A significant impairment in emotion recognition and empathy was detected in both the classical and overlap FTD syndromes. The recognition of positive emotions was relatively preserved compared to that of negative emotions. Among the FTD subtypes, maximal impairment of empathy was demonstrated in FTD-PSP. Conclusion: Social cognition impairment is
\end{abstract}


pervasive across the spectrum of FTD disorders, and tests of emotion recognition and empathy are clinically useful to identify the nature of behavioural problems in both classical and overlap FTD. Our findings also have implications for understanding the neural basis of social cognition in FTD.

\section{Introduction}

Frontotemporal dementia (FTD) syndromes are a complex group of neurodegenerative disorders characterised by profound changes in behaviour and cognition [1]. The FTD spectrum is heterogeneous and comprises classical behavioural-variant (Bv)FTD and the language variant primary progressive aphasia (PPA), which includes progressive non-fluent aphasia (PNFA) and semantic dementia (SD) [2,3]. Recently, overlap syndromes are increasingly being recognized; these are characterised by a range of motor features in association with the cognitive-behavioural syndrome of FTD. These include: FTD amyotrophic lateral sclerosis (FTD-ALS), FTD progressive supranuclear palsy (FTD-PSP), and FTD corticobasal syndrome (FTD-CBS) [4-8]. Research indicates that social cognition deficits underlie the complex behavioural problems exhibited by patients with FTD [9]. Social cognition refers to the processing of social information and is conceptually categorised into 3 domains: emotion recognition, empathy, and theory of mind [10]. Emotion recognition is the ability to identify another person's emotions. The ability to understand, be aware of, and vicariously experience the feelings, thoughts, and experience of another person constitute empathy; theory of mind is the ability to attribute independent mental states to other individuals in order to understand and predict their behaviour [11].

While social cognition deficits contribute significantly to disability and care-giver stress, they are often underdiagnosed [12]. The importance of assessing social cognition is now formally recognized in the 5th edition of the Diagnostic and Statistical Manual (DSM-5) criteria for Mental Disorders, and it is included as a core neurocognitive domain to diagnose dementia [13]. The standard tests used in the assessment of emotion recognition include pictures of facial affect (POFA) [14], the Florida Affect Battery [15], and the Interpersonal Reactivity Index (IRI) to evaluate empathy [16].

Social cognition impairment has been consistently demonstrated in BvFTD [1, 7-9, 17-21]. Impaired emotion recognition and empathy have also been reported in patients with PPA [22-26]. Similar investigations into overlap FTD syndromes are very limited, and these studies have small sample sizes and use variable methods to identify social cognition deficits with varying results [27-29]. The nature and extent of social cognition deficits across the different subtypes of FTD is hence still unclear. Our study was designed to investigate the frequency and pattern of the social cognition deficits, emotion recognition and empathy, in classical (BvFTD, PNFA, and SD) and overlap FTD (FTD-ALS and FTD-PSP) syndromes, using tests that are locally validated and clinically relevant.

\section{Methodology}

Participants

Of the 75 consecutive FTD patients evaluated in the Cognitive Neurology Clinic during the study period 2017-2019, social cognition was assessed in 60 patients. Fifteen patients were excluded due to severe disease (Clinical Dementia Rating Scale [CDR] score $\leq 2$ ), non-availability of reliable caregiver or incomplete data. The sample size of 60 was considered adequate 
to assess social cognition in the dementia cohort according to a power analysis calculation (a power of 0.81 at an $\alpha$ value of 0.05 ). The classical FTD syndromes included 16 cases of BvFTD and 20 of PPA (13 PNFA and 7 SD). The overlap FTD syndromes included 13 cases of FTD-PSP and 11 of FTD-ALS. The diagnoses of BvFTD, PNFA, and SD were based on the Frontotemporal Lobar Degeneration Criteria [30]. The diagnosis of FTD-PSP was based on the criteria in Litvan et al. [4]. The diagnosis of FTD-ALS was based on the criteria in Strong et al. [31]. The diagnosis of overlap FTD syndromes was made in patients who had features of both classical FTD (either behavioural or language) and motor features (ALS or PSP) at the time of presentation.

All 13 patients with FTD-PSP had both behavioural symptoms and features of PSP at their initial evaluation. In 9 of these 13, the onset was with behavioural features, 3 patients showed behavioural abnormalities that followed features of PSP, and 1 patient with FTD-PSP had additional features of PNFA. Of the 11 patients with overlap FTD-ALS, 6 had features satisfying the consensus criteria for BvFTD at the onset of illness and subsequently developed manifestations of ALS at presentation, but the onset of these 2 symptom complexes was in the reverse order in the other 5 patients.

Demographic and clinical data was collected through interviews with patients and reliable caregivers. All patients were subjected to neurological and neuropsychological evaluations, laboratory tests, and brain MRI. Cognitive assessment was done using Addenbrooke's Cognitive Examination-III (ACE-III) [32] adapted for Indian languages. The Frontal Systems Behavioural Evaluation (FrSBe) [33] was used to evaluate behaviour. The neuropsychological evaluation was done using Digit Span and the Trail-Making Test A \& B (TMT A \& B) for attention and executive functions [34], the Verbal Learning Test (VLT) and modified Taylor Complex Figure Test (MTCF) for episodic memory, the Picture Naming Test (PNT) for language assessment, and an MTCF copy was used for the assessment of visuospatial functions. These tests were derived from the Indian Council of Medical Research Neurocognitive Tool Box (the ICMR-NCTB), which is adapted and validated for use in Indian languages [35]. Semantic memory was assessed using a semantic association task, a subtask of the Indian version of the Cambridge Semantic Battery [36].

\section{Measures of Social Cognition}

Social cognition was assessed in all patients using the IRI for empathy and POFA for emotion recognition [37]. These tests have been adapted and validated for the Indian context [34-36].

Interpersonal Reactivity Index

The IRI, a test of empathy, has good psychometric properties across various cultures [16]. It includes 4 categories: perspective-taking (PT), empathic concern (EC), personal distress (PD), and fantasy scale (FS), with 7 items in each category giving a total of 28 items. Each item is answered using a 5-point Likert scale ranging from "does not describe him/her well" to "describes him/her very well". Pre- and post-disease onset scores were collected. PT measures the tendency to spontaneously adopt the psychological point of view of others in everyday life. Similarly, EC measures the extent to which an individual experiences sympathy for others. FS evaluates an individual's ability to switch himself/herself into the roles of fictitious characters in books or movies. PD measures "self-oriented" feelings of personal anxiety in tense interpersonal settings.

Pictures of Facial Affect

POFA was used to examine the ability to identify emotions according to emotional valence of faces. The original test [14] consisted of 110 black-and-white photographs of facial expres-

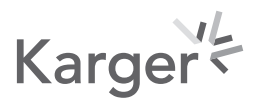


Arshad et al.: Social Cognition Deficits in Frontotemporal Dementia Spectrum Disorders

Table 1. Demographic and clinical characteristics of the subtypes of frontotemporal dementia (FTD)

\begin{tabular}{|c|c|c|c|c|c|c|}
\hline & $\begin{array}{l}\text { BvFTD } \\
(n=16)\end{array}$ & $\begin{array}{l}\text { PNFA } \\
(n=13)\end{array}$ & $\begin{array}{l}\text { SD } \\
(n=7)\end{array}$ & $\begin{array}{l}\text { FTD-ALS } \\
(n=11)\end{array}$ & $\begin{array}{l}\text { FTD-PSP } \\
(n=13)\end{array}$ & $p$ value \\
\hline Age, years & $56.1(9.8)$ & $60.3(5.2)$ & $58.5(5.9)$ & $59.5(6.1)$ & $63.4(8.1)$ & 0.153 \\
\hline Years of education & $11.8(4.5)$ & $9.0(5.0)$ & $11.4(4.6)$ & $9.8(2.6)$ & $8.1(5.5)$ & 0.234 \\
\hline Males, $n(\%)$ & $10(62.5)$ & $8(61.5)$ & $4(57.1)$ & $9(81.8)$ & $6(46.2)$ & 0.512 \\
\hline Duration of symptoms, months & $40.0(39.0)$ & $30.0(28.8)$ & $35.1(13.1)$ & $28.1(14.4)$ & CDR score, $\%$ & 0.785 \\
\hline Very mild & & & & & $2(16.7)$ & 0.117 \\
\hline Mild & $6(46.2)$ & $2(33.3)$ & $5(38.5)$ & $8(50.0)$ & $8(66.7)$ & \\
\hline Moderate & $7(53.8)$ & $5(66.7)$ & $8(61.5)$ & $8(50.0)$ & $2(16.7)$ & \\
\hline \multicolumn{7}{|l|}{ ACE-III } \\
\hline Total score (max score: 100 ) & $48.6(20.9)$ & $39.5(17.1)$ & $52.3(24.7)$ & $53.4(14.8)$ & $47.4(22.9)$ & 0.481 \\
\hline \multicolumn{7}{|l|}{ ACE-III subscale scores } \\
\hline Attention (max score: 18 ) & $9.1(4.5)$ & $12.0(4.9)$ & $8.8(3.9)$ & $10.6(5.1)$ & $11.1(3.5)$ & 0.449 \\
\hline Memory (max score: 26) & $12.0(4.9)$ & $8.8(3.9)$ & $10.6(5.1)$ & $11.1(3.5)$ & $9.1(4.5)$ & 0.399 \\
\hline Fluency (max score: 14) & $4.9(2.9)$ & $1.9(2.1)$ & $4.1(3.2)$ & $5.3(3.3)$ & $6.7(2.8)$ & $0.003^{\mathrm{a}}$ \\
\hline Language (max score: 26 ) & $12.0(7.5)$ & $9.4(7.1)$ & $6.5(6.1)$ & $9.9(5.1)$ & $8.9(5.2)$ & $0.246^{\mathrm{b}}$ \\
\hline Visuospatial (max score: 16) & $9.7(5.2)$ & $11.6(4.5)$ & $9.9(3.8)$ & $10.7(4.5)$ & $11.4(2.5)$ & 0.783 \\
\hline
\end{tabular}

Values are represented as mean (standard deviation), unless otherwise indicated. BvFTD, behavioural-variant frontotemporal dementia; PNFA, progressive non-fluent aphasia; SD, semantic dementia; ALS, amyotrophic lateral sclerosis; PSP, progressive supranuclear palsy; CDR, Clinical Dementia Rating Scale; ACE-III, Addenbrooke's Cognitive Examination-III.

${ }^{a}$ Post hoc analysis showed significantly reduced fluency in PNFA compared to BvFTD, FTD-ALS, and FTD-PSP.

b SD had significantly impaired language compared to BvFTD.

sions. Happiness and surprise are considered as positive emotions while anxiety, disgust, fear, and sadness are considered as negative emotions. Keeping in view the lower attentionspan for testing in dementia patients, we adapted a shorter version comprising 56 pictures, with 8 pictures for each emotion [36]. The percentage of correct responses for each emotion was calculated.

\section{Statistical Analysis}

All data was analysed using the Statistical Package for Social Sciences (SPSS) software v16.0 (SPSS, Chicago, IL, USA). The demographic comparison of continuous variables was done using ANOVA and categorical variables by means of the $\chi^{2}$ or Fisher's exact test. The performance in neuropsychological tests, FrSBe and IRI scores before and after illness, and POFA score were assessed using ANOVA, and Tukey's test was used for post hoc analysis of normally distributed data. The Kruskal-Wallis test was used to analyse the non-normally distributed data (TMT A \& B and MTCF). The missing values were not considered for the ANOVA analysis. The paired $t$ test was used to compare IRI and FrSBe scores pre- and postillness between different FTD subtypes. The correlation between ACE-III and POFA scores were determined by Pearson's/Spearman's rank correlation. $p<0.05$ was considered statistically significant.

\section{Results}

Demographic and Clinical Characteristics

A total of 60 patients with classical and overlap FTD syndromes were recruited. Mean age of the cohort was $59.5 \pm 7.8$ years, mean number of years of education was $10.0 \pm 4.7,61 \%$ 
Arshad et al.: Social Cognition Deficits in Frontotemporal Dementia Spectrum Disorders

Table 2. Neuropsychological test performance in subtypes of frontotemporal dementia (FTD)

\begin{tabular}{|c|c|c|c|c|c|c|}
\hline & $\begin{array}{l}\text { BvFTD } \\
(n=16)\end{array}$ & $\begin{array}{l}\text { PNFA } \\
(n=13)\end{array}$ & $\begin{array}{l}\text { SD } \\
(n=7)\end{array}$ & $\begin{array}{l}\text { FTD-ALS } \\
(n=11)\end{array}$ & $\begin{array}{l}\text { FTD-PSP } \\
(n=13)\end{array}$ & $p$ value \\
\hline \multicolumn{7}{|l|}{ Attention and executive function } \\
\hline Digit forward $(6 \pm 1)$ & $4.1(1.9)$ & $3.0(1.2)$ & $3.6(1.3)$ & $4.0(1.1)$ & $3.0(1.6)$ & 0.138 \\
\hline Digit backward $(5 \pm 1)$ & $2.7(1.8)$ & $1.5(1.5)$ & $2.0(1.5)$ & $2.8(1.4)$ & $2.31(1.8)$ & 0.244 \\
\hline Trail-Making Test A (time in seconds) & $276.0(132.2)$ & $225.9(146.1)$ & $72.0(8.5)$ & $198.0(155.2)$ & $139.2(98.4)$ & 0.231 \\
\hline Trail-Making Test B (time in seconds) & $419.1(215.5)$ & $287.1(266.0)$ & $162.0(42.4)$ & $340.5(188.7)$ & $290.4(226.7)$ & 0.634 \\
\hline \multicolumn{7}{|l|}{ Episodic memory } \\
\hline VLT total recall (max score: 30 ) & $10.1(5.4)$ & $9.5(5.6)$ & $7.6(4.9)$ & $11.8(7.9)$ & $8.7(4.8)$ & 0.400 \\
\hline VLT delayed recall (max score: 10 ) & $2.0(2.1)$ & $1.7(1.9)$ & $2.7(2.1)$ & $3.0(2.7)$ & $2.2(2.0)$ & 0.507 \\
\hline VLT delayed recognition (max score: 10 ) & $5.5(3.3)$ & $5.1(3.4)$ & $5.0(3.3)$ & $4.7(3.4)$ & $4.2(3.8)$ & 0.944 \\
\hline \multicolumn{7}{|l|}{ Language and semantic memory } \\
\hline PNT (max score: 120$)$ & $76.2(37.2)$ & $60.5(29.4)$ & $57.6(37.5)$ & $70.0(31.6)$ & $22.3(11.1)$ & $0.021^{*}$ \\
\hline Semantic association test (max score: 42 ) & $24.8(14.9)$ & $29.3(11.3)$ & $26.0(13.3)$ & $33.3(8.5)$ & $7.8(3.7)$ & $0.002^{*}$ \\
\hline \multicolumn{7}{|l|}{ Visuospatial tests } \\
\hline MTCF test copy (max score: 36 ) & $21.9(13.7)$ & $22.6(12.2)$ & $22.0(17.3)$ & $18.8(9.3)$ & $26.5(12.0)$ & 0.964 \\
\hline MTCF test immediate recall (max score: 36 ) & $6.9(10.9)$ & $6.4(7.7)$ & $12.7(10.1)$ & $7.8(6.6)$ & $13.5(19.1)$ & 0.809 \\
\hline MTCF test recall (max score: 36 ) & $4.7(7.6)$ & $2.9(2.3)$ & $5.0(7.0)$ & $2.0(2.8)$ & $8.0(11.3)$ & 0.364 \\
\hline
\end{tabular}

Values are represented as mean (standard deviation). BvFTD, behavioural-variant frontotemporal dementia; PNFA, progressive non-fluent aphasia; SD, semantic dementia; ALS, amyotrophic lateral sclerosis; PSP, progressive supranuclear palsy; MCTF, modified Taylor Complex Figure Test; VLT, Verbal Learning test; PNT, Picture-Naming Test.

* Post hoc analysis showed significantly impaired PNT and semantic association task in SD compared to the other subtypes.

were males, and the mean duration of symptoms was $34.1 \pm 4.8$ months. Among the 60 patients, $16.6 \%$ (10) had a family history of memory, behavioural, or motor disturbances. There was no difference between the subtypes in demographic profile, age at presentation, duration of symptoms, and dementia severity (Table 1 ).

\section{Cognitive Characteristics of the FTD Subtypes}

The mean total scores on ACE-III were comparable across all subtypes (Table 1). With respect to the subdomains of ACE-III, fluency was lowest in PNFA versus BvFTD $(p<0.003)$, FTD-ALS $(p<0.001)$, and FTD-PSP $(p<0.010)$. Language scores were significantly lower in $\mathrm{SD}$ than in BVFTD $(p<0.039)$. In the neuropsychological evaluation, tests of attention, executive function, and episodic memory were equally impaired across all subgroups (Table 2). The SD cohort had the poorest performance of all the subtypes in the PNT $(p<0.021)$ and semantic association task $(p<0.002)$. With respect to the assessment of executive and visuospatial functions, $13(21.6 \%)$ and $12(20 \%)$ patients were not able to perform the TMT A \& B and MTCF tests, respectively, due to behavioural disturbances, impaired comprehension, or motor impairment. No significant differences were found between subtypes among the patients tested.

\section{Behavioural Features of the FTD Subtypes}

Caregivers of the entire FTD cohort as well the individual subtypes reported a significant change across all behaviours: apathy $\left(t_{59}=-12.2 ; p<0.001 ; 95 \%\right.$ confidence interval [CI] -21.9 to -15.8$)$, disinhibition ( $t_{59}=-8.4 ; p<0.001 ; 95 \%$ CI -15.5 to -9.6 ), and executive dysfunction $\left(t_{59}=-4.6 ; p<0.001 ; 95 \% \mathrm{CI}-10.8\right.$ to -4.6$)$. There were no differences noted between the different subtypes of FTD (Table 3). 
Arshad et al.: Social Cognition Deficits in Frontotemporal Dementia Spectrum Disorders

Table 3. Mean FrSBe $t$ scores for subtypes of frontotemporal dementia (FTD) pre- and post-illness

\begin{tabular}{lcccc}
\hline & Pre-illness & Post-illness & Mean difference & $p$ value \\
\hline Apathy & & & & \\
$\quad$ Entire FTD cohort $(n=60)$ & $58.5(7.2)$ & $77.5(11.9)$ & $18.9(11.9)$ & $<0.001$ \\
BvFTD $(n=16)$ & $58.5(7.8)$ & $80.5(14.7)$ & $22.0(13.7)$ & $<0.001$ \\
PNFA $(n=13)$ & $58.6(5.9)$ & $77.9(11.2)$ & $19.3(13.3)$ & $<0.001$ \\
SD $(n=7)$ & $61.4(9.2)$ & $72.8(9.5)$ & $11.4(5.1)$ & $<0.001$ \\
FTD-ALS $(n=11)$ & $59.1(5.1)$ & $79.4(14.3)$ & $20.3(12.9)$ & $<0.001$ \\
FTD-PSP $(n=13)$ & $56.3(8.0)$ & $74.0(7.0)$ & $19.3(13.3)$ & $<0.001$ \\
Disinhibition & & & & \\
Entire FTD cohort $(n=60)$ & $55.6(9.6)$ & $68.2(13.3)$ & $12.6(11.5)$ & $<0.001$ \\
BvFTD $(n=16)$ & $55.1(6.7)$ & $69.3(10.0)$ & $14.3(7.2)$ & $<0.001$ \\
PNFA $(n=13)$ & $59.3(10.8)$ & $68.0 .5(11.9)$ & $8.6(11.9)$ & 0.022 \\
SD $(n=7)$ & $55.4(11.6)$ & $68.7(8.2)$ & $13.3(10.4)$ & 0.015 \\
FTD-ALS $(n=11)$ & $53.7(5.9)$ & $70.9(22.8)$ & $17.1(16.6)$ & 0.006 \\
FTD-PSP $(n=13)$ & $54.2(8.7)$ & $64.2(10.1)$ & $10.0(10.8)$ & 0.006 \\
Executive dysfunction & & & & \\
Entire FTD cohort $(n=60)$ & $58.1(7.5)$ & $65.9(10.4)$ & $7.7(12.0)$ & $<0.001$ \\
BvFTD $(n=16)$ & $57.5(10.7)$ & $65.6(10.7)$ & $8.1(13.0)$ & 0.026 \\
PNFA $(n=13)$ & $57.3(8.4)$ & $67.2(13.9)$ & $9.9(16.7)$ & 0.053 \\
SD $(n=7)$ & $61.0(6.7)$ & $67.0(7.0)$ & $18.3(15.3)$ & 0.019 \\
FTD-ALS $(n=11)$ & $55.9(5.7)$ & $66.9(11.3)$ & $11.0(11.3)$ & 0.009 \\
FTD-PSP $(n=13)$ & $60.1(9.0)$ & $63.4(7.0)$ & $3.3(8.4)$ & 0.018 \\
Total FrSBE score & $115.7(19.9)$ & $132.1(21.0)$ & $16.4(10.6)$ & $<0.001$ \\
\hline
\end{tabular}

Values are represented as mean (standard deviation). BvFTD, behavioural-variant frontotemporal dementia; PNFA, progressive non-fluent aphasia; SD, semantic dementia; ALS, amyotrophic lateral sclerosis; PSP, progressive supranuclear palsy; FrSBE, Frontal System Behavioural Evaluation.

\section{Assessment of Social Cognition}

The IRI scores showed significant changes in 3 empathy domains pre- and post-illness: PT $\left(t_{59}=12.6, p<0.001 ; 95 \%\right.$ CI 5.3-7.4), EC ( $t_{59}=8.7, p<0.001 ; 95 \%$ CI 7.3-10.1) and PD $\left(t_{59}=1.1, p<0.038 ; 95 \% \mathrm{CI}-2.0\right.$ to -0.1$)$ in the whole FTD cohort. There was no difference in the change in FS score $\left(t_{59}=0.7, p=0.464 ; 95 \% \mathrm{CI}-0.5\right.$ to 1.1$)$. BvFTD, SD, FTD-ALS, and FTD-PSP showed a significant decline in PT and EC scores after illness (Table 4). On comparison of the magnitude of change in empathy, EC scores deteriorated the most in FTD-PSP $(11.6 \pm 5.9)$ when compared to the other subtypes (BvFTD $8.7 \pm 5.4$, PNFA $10.2 \pm$ 4.8, SD $5.5 \pm 3.6$, FTD-ALS $6.6 \pm 5.9 ; F_{4,55}=2.93 ; p=0.029$; partial $\eta^{2}=0.21$ ).

On analysis of emotion recognition in classical and overlap FTD syndromes, happy was the most recognised emotion $(78.9 \pm 22.3)$ while scores for disgust $(19.4 \pm 22.0)$, fear $(11.9 \pm$ $16.9)$, and anxiety $(13.3 \pm 19.4)$ were the lowest in the whole cohort $(p<0.001)$. There were no significant differences between subtypes of FTD in recognising most emotions (Table 5). A difference was noted only for surprise: the BvFTD group was relatively less impaired than the other subtypes $(p=0.035)$.

A positive correlation between ACE-III, POFA total score, and the pre- and post-disease onset empathy scores of PT and EC on IRI was observed across the whole FTD cohort $(r=0.6$, $p<0.001 ; r=0.4, p<0.001 ; r=0.5, p<0.001$ ). 
Table 4. Interpersonal Reactivity Index (IRI) scores before and after illness for subtypes of frontotemporal dementia (FTD)

\begin{tabular}{|c|c|c|c|c|}
\hline & Before illness & After illness & Mean difference & $p$ value \\
\hline \multicolumn{5}{|l|}{ Perspective-taking } \\
\hline Entire FTD cohort $(n=60)$ & $18.3(4.6)$ & $11.9(4.5)$ & $6.4(3.9)$ & $<0.001$ \\
\hline $\operatorname{BvFTD}(n=16)$ & $17.7(4.4)$ & $11.9(4.9)$ & $5.8(3.7)$ & $<0.001$ \\
\hline PNFA $(n=13)$ & $19.1(5.0)$ & $12.2(5.6)$ & $6.9(4.6)$ & 0.377 \\
\hline $\operatorname{SD}(n=7)$ & $21.5(4.9)$ & $12.0(2.8)$ & $8.1(5.2)$ & $<0.001$ \\
\hline FTD-ALS $(n=11)$ & $17.7(4.6)$ & $14.0(3.5)$ & $3.7(2.4)$ & 0.074 \\
\hline FTD-PSP $(n=13)$ & $17.6(3.6)$ & $9.9(3.9)$ & $7.7(2.8)$ & $<0.001$ \\
\hline \multicolumn{5}{|l|}{ Fantasy scale } \\
\hline Entire FTD cohort $(n=60)$ & $5.1(4.0)$ & $4.8(4.6)$ & $0.3(2.9)$ & 0.464 \\
\hline $\operatorname{BvFTD}(n=16)$ & $4.8(3.1)$ & $5.8(5.6)$ & $-1.0(4.4)$ & 0.377 \\
\hline PNFA $(n=13)$ & $7.2(4.8)$ & $5.5(4.7)$ & $1.7(2.7)$ & 0.041 \\
\hline $\operatorname{SD}(n=7)$ & $4.7(2.7)$ & $3.8(2.9)$ & $0.6(1.3)$ & 0.280 \\
\hline FTD-ALS $(n=11)$ & $4.9(5.9)$ & $4.5(5.9)$ & $0.5(1.3)$ & 0.211 \\
\hline FTD-PSP $(n=13)$ & $3.7(2.2)$ & $3.6(2.4)$ & $0.2(2.5)$ & 0.827 \\
\hline \multicolumn{5}{|l|}{ Empathy concern } \\
\hline Entire FTD cohort $(n=60)$ & $20.4(4.9)$ & $11.7(4.8)$ & $8.7(5.4)$ & $<0.001$ \\
\hline $\operatorname{BvFTD}(n=16)$ & $20.9(5.1)$ & $10.7(5.4)$ & $10.1(4.7)$ & $<0.001$ \\
\hline PNFA $(n=13)$ & $19.8(4.5)$ & $12.0(4.4)$ & $7.8(5.3)$ & $<0.001$ \\
\hline $\operatorname{SD}(n=7)$ & $20.7(2.7)$ & $12.2(2.3)$ & $6.6(5.9)$ & 0.025 \\
\hline FTD-ALS $(n=11)$ & $19.6(5.1)$ & $14.1(2.6)$ & $5.5(3.6)$ & $<0.001$ \\
\hline FTD-PSP $(n=13)$ & $21.7(5.8)$ & $10.1(5.9)$ & $11.6(5.9)$ & $<0.001$ \\
\hline \multicolumn{5}{|l|}{ Personal distress } \\
\hline Entire FTD cohort $(n=60)$ & $9.8(3.9)$ & $10.9(4.2)$ & $-1.1(3.9)$ & 0.038 \\
\hline $\operatorname{BvFTD}(n=16)$ & $9.6(3.6)$ & $12.0(4.7)$ & $-2.4(4.9)$ & 0.074 \\
\hline PNFA $(n=13)$ & $10.5(4.4)$ & $11.5(4.1)$ & $-1.1(3.8)$ & 0.324 \\
\hline $\operatorname{SD}(n=7)$ & $7.7(3.4)$ & $8.5(1.2)$ & $-0.6(2.3)$ & 0.522 \\
\hline FTD-ALS $(n=11)$ & $10.6(3.6)$ & $12.1(4.9)$ & $-1.5(3.1)$ & 0.152 \\
\hline FTD-PSP $(n=13)$ & $9.3(4.2)$ & $8.6(2.7)$ & $-0.7(3.3)$ & 0.461 \\
\hline
\end{tabular}

Values are represented as mean (standard deviation). BvFTD, behavioural-variant frontotemporal dementia; PNFA, progressive non-fluent aphasia; SD, semantic dementia; ALS, amyotrophic lateral sclerosis; PSP, progressive supranuclear palsy.

\section{Discussion}

This study explored social cognition, an underrecognised cognitive domain, across the whole spectrum of FTD syndromes. A noteworthy finding was the consistent impairment in empathy and emotion recognition in not only the classical, but also the overlap syndromes of FTD. A differential pattern of deficits emerged with regard to social cognition in the subtypes. Maximum deficits in empathy were noticed in FTD-PSP, which is a novel finding. Our findings emphasise the importance of evaluating social cognition in both classical (BvFTD, PNFA, and SD) and overlap FTD syndromes (FTD-ALS and FTD-PSP), and offer insights into understanding the basis of behavioural disturbances in FTD syndromes.

All the subtypes of FTD were comparable for their general cognitive functioning, as demonstrated by the ACE-III scores. Evaluation of individual cognitive domains revealed patterns typical of the diagnosed FTD syndrome. Consistent with the diagnosis of the language variant of FTD, fluency scores were disproportionately lowest in PNFA patients, and SD patients showed gross impairment of semantic memory and language [38, 39]. Executive functions were comparably impaired across both classical and overlap syndromes [40-44] and visuospatial functions were mildly impaired in all subtypes [45]. 
Arshad et al.: Social Cognition Deficits in Frontotemporal Dementia Spectrum Disorders

Table 5. Emotion recognition scores in the subtypes of frontotemporal dementia (FTD)

\begin{tabular}{|c|c|c|c|c|c|c|}
\hline & $\begin{array}{l}\text { BvFTD } \\
(n=16)\end{array}$ & $\begin{array}{l}\text { PNFA } \\
(n=13)\end{array}$ & $\begin{array}{l}\text { SD } \\
(n=7)\end{array}$ & $\begin{array}{l}\text { FTD-ALS } \\
(n=11)\end{array}$ & $\begin{array}{l}\text { FTD-PSP } \\
(n=13)\end{array}$ & $p$ value \\
\hline PoFA-happy & $84.4(19.6)$ & $76.9(20.3)$ & 82.1 (22.7) & $88.6(8.8)$ & $64.4(29.2)$ & 0.059 \\
\hline POFA-sad & $42.9(25.4)$ & $44.2(26.3)$ & $30.4(27.8)$ & $54.6(29.1)$ & $45.2(24.8)$ & 0.464 \\
\hline POFA-surprise & $42.9(34.5)$ & $17.3(19.5)$ & $14.3(27.4)$ & $14.8(23.6)$ & $20.2(25.3)$ & $0.035^{*}$ \\
\hline POFA-neutral & $43.8(30.6)$ & $46.2(34.4)$ & $57.1(35.3)$ & $32.9(25.5)$ & $24.0(23.6)$ & 0.127 \\
\hline POFA-disgust & $28.9(24.5)$ & $23.1(22.7)$ & $14.3(23.3)$ & $17.1(21.1)$ & 8.7 (13.9) & 0.135 \\
\hline POFA-fear & $17.9(22.8)$ & 11.5 (12.9) & $10.7(19.7)$ & $10.2(15.6)$ & $6.7(10.9)$ & 0.506 \\
\hline POFA-anxiety & $19.5(22.8)$ & $9.6(11.6)$ & $5.4(9.8)$ & $17.1(25.2)$ & $10.6(18.9)$ & 0.420 \\
\hline POFA-total & $40.1(16.7)$ & 32.7 (10.9) & $30.4(15.4)$ & $34.4(15.4)$ & $26.1(14.6)$ & 0.157 \\
\hline
\end{tabular}

Values are represented as mean (standard deviation). BvFTD, behavioural-variant frontotemporal dementia; PNFA, progressive non-fluent aphasia; SD, semantic dementia; ALS, amyotrophic lateral sclerosis; PSP, progressive supranuclear palsy; POFA, pictures of facial affect.

* Post hoc analysis showed that BvFTD patients recognised the surprise emotion significantly better than PNFA, SD, FTD-ALS, and FTD-PSP.

Apathy and executive dysfunction were prominent in the entire cohort as revealed by the FrSBe scores. These behavioural changes are typical of classical FTD syndromes [40, 46, 47]. Disinhibition was found in almost the same proportion across all the subtypes. While disinhibited behaviour is considered a typical feature of BvFTD [48-50], a few studies report disinhibition also with SD, and to a lesser extent in PNFA patients [46, 51]. The frequency of disinhibition in overlap syndromes, i.e., FTD-ALS and FTD-PSP, has not been previously reported. The predominant regions of the brain implicated in behavioural control include the anterior cingulate and orbitofrontal areas [52], typically known to be involved in BvFTD. Our findings suggest that, regardless of the FTD subtype, the disease process is likely to involve inhibitory control circuits in the brain, even in the overlap FTD syndromes.

Two domains of social cognition, i.e., empathy and emotion recognition, were evaluated. On investigating the pattern of deficits in empathy, we found that empathic deficits occur not only in patients with BvFTD, but also in the language variants. Loss of empathy is found to be a central feature of BvFTD [53-55], and while disturbance of empathy has been reported in SD and PNFA, the actual extent to which it is impaired is as yet unclear, mainly due to the small number of studies and the variable results [54-57]. Empathy in FTD-ALS has also not yet been systematically evaluated. In our cohort, patients with FTD-ALS were significantly impaired in empathic concern. Empathic disturbances have been explored in only small groups of patients with FTD-PSP, with one study showing impairment in emotional empathy [58] and the other demonstrating variability in PT [55]. In our group of patients with FTD-PSP, both PT and EC were impaired, and the severity of the decline in EC was maximal in this group of patients. Empathy in FTD patients corresponds to multiple areas in the brain including the frontoinsular and temporal structures [27, 59]. The finding of impaired EC in the overlap syndromes FTD-ALS and FTD-PSP is significant and may reflect the deterioration of these brain areas and networks early in these diseases. The maximum deterioration observed in FTD-PSP is supported by imaging data that implicate a wide network of areas in PSP that include the insular and frontomedian regions, in addition to the striatum and brainstem areas [60].

Emotion recognition deficits were demonstrable in the language variants PNFA and SD, similar to BvFTD, and consistent with the reported literature [21-24]. However, emotion recognition in the overlap FTD syndromes has not ever received much attention $[27,59]$. In 
our study, the overlap syndromes, FTD-ALS and FTD-PSP, were also found to demonstrate impairment of emotion recognition. A differential pattern of recognition is known to exist between the different emotions: happy emotions are better recognised than negative emotions $[1,24,28,61,62]$, and our findings confirm this. This dissociation could be due to the preservation of positive emotions in the early stage of disease $[1,18]$.

There are a few limitations to the study. Although the overall sample size was quite large, individual subtypes had smaller numbers. While we explored emotion recognition and empathy, the domain of theory of mind has still to be explored. Functional imaging and genetic testing were not done as a part of this study. Larger numbers of patients and the addition of tests of theory of mind will further validate our findings.

To conclude, the study established the relevance of clinically evaluating the domain of social cognition across the entire FTD spectrum and demonstrated that emotion recognition and empathy were clearly impaired in both classical and overlap FTD syndromes. This emphasises the need to clinically evaluate FTD syndromes with tests of social cognition, regardless of their subtype. The testing of social cognition assumes importance in characterising disease profile for the proper management and counselling of caregivers.

\section{Acknowledgements}

We would like to thank Shanta G. for her help in validation of social cognition tests.

\section{Statement of Ethics}

The study was approved by the Institutional Ethics Committee of NIMHANS and all participants provided a written consent. The research was conducted ethically in accordance with the World Medical Association Declaration of Helsinki.

\section{Conflict of Interest Statement}

A.P. is funded by Department of Science and Technology - Science and Engineering Research Board (DST-SERB). F.V. is funded by the Indian Council of Medical Research. The authors declare no competing interests.

\section{Funding Sources}

The authors report no specific funding in relation to this research.

\section{Author Contributions}

F.A.: acquisition of data, analysis, and interpretation of data, and drafting of the manuscript. A.P.: analysis and interpretation of data, drafting and critical revision of the manuscript for intellectual content. S.M.: statistical analysis and interpretation of data and critical revision of the manuscript for intellectual content. F.V.: statistical analysis, interpretation of findings, and revision of the manuscript. V.V.P., D.J.K., and L.S.: analysis and interpretation of data, and revising the manuscript for content. S.V., R.Y., P.K.P., and A.N.: analysis and interpretation of 
data, revising the manuscript for content, and study supervision. S.A.: study concept and design, analysis and interpretation of data, drafting/revising the manuscript for intellectual content, study supervision and coordination, obtaining funding. All authors reviewed the final manuscript.

\section{References}

1 Lavenu I, Pasquier F, Lebert F, Petit H, Van der Linden M. Perception of emotion in frontotemporal dementia and Alzheimer disease. Alzheimer Dis Assoc Disord. 1999 Apr-Jun;13(2):96-101.

2 Rascovsky K, Hodges JR, Knopman D, Mendez MF, Kramer JH, Neuhaus J, et al. Sensitivity of revised diagnostic criteria for the behavioural variant of frontotemporal dementia. Brain. 2011 Sep;134(Pt 9):2456-77.

3 Gorno-Tempini ML, Hillis AE, Weintraub S, Kertesz A, Mendez M, Cappa SF, et al. Classification of primary progressive aphasia and its variants. Neurology. 2011 Mar;76(11):1006-14.

4 Litvan I, Agid Y, Calne D, Campbell G, Dubois B, Duvoisin RC, et al. Clinical research criteria for the diagnosis of progressive supranuclear palsy (Steele-Richardson-Olszewski syndrome): report of the NINDS-SPSP international workshop. Neurology. 1996 Jul;47(1):1-9.

5 Armstrong MJ, Litvan I, Lang AE, Bak TH, Bhatia KP, Borroni B, et al. Criteria for the diagnosis of corticobasal degeneration. Neurology. 2013 Jan;80(5):496-503.

6 Burrell JR, Halliday GM, Kril JJ, Ittner LM, Götz J, Kiernan MC, et al. The frontotemporal dementia-motor neuron disease continuum. Lancet. 2016 Aug;388(10047):919-31.

7 Alladi S, Mekala S, Chadalawada SK, Jala S, Mridula R, Kaul S. Subtypes of dementia: a study from a memory clinic in India. Dement Geriatr Cogn Disord. 2011;32(1):32-8.

8 Murley AG, Coyle-Gilchrist I, Rouse MA, Jones PS, Li W, Wiggins J, et al. Redefining the multidimensional clinical phenotypes of frontotemporal lobar degeneration syndromes. Brain. 2020 May;143(5):1555-71.

9 Lough S, Kipps CM, Treise C, Watson P, Blair JR, Hodges JR. Social reasoning, emotion and empathy in frontotemporal dementia. Neuropsychologia. 2006;44(6):950-8.

10 Henry JD, von Hippel W, Molenberghs P, Lee T, Sachdev PS. Clinical assessment of social cognitive function in neurological disorders. Nat Rev Neurol. 2016 Jan;12(1):28-39.

11 Premack D, Woodruff G. Does the chimpanzee have a theory of mind? Behav Brain Sci. 1978;1(4):515-26.

12 Kaizik C, Caga J, Camino J, O'Connor CM, McKinnon C, Oyebode JR, et al. Factors underpinning caregiver burden in frontotemporal dementia differ in spouses and their children. J Alzheimers Dis. 2017;56(3):1109-17.

13 Regier DA, Kuhl EA, Kupfer DJ. The DSM-5: classification and criteria changes. World Psychiatry. 2013 Jun; 12(2):92-8.

14 Ekman P, Friesen WV. Pictures of Facial Affect. Palo Alto (CA): Consulting Psychologists Press; 1976. pp. 1-8.

15 Bowers D, Blonder LX, Heilman KM. Florida affect battery. Florida, USA: Center for Neuropsychological Studies, Department of Neurology; 1998.

16 Davis MH. Measuring individual differences in empathy: evidence for a multidimensional approach. J Pers Soc Psychol. 1983;44(1):113-26.

17 Keane J, Calder AJ, Hodges JR, Young AW. Face and emotion processing in frontal variant frontotemporal dementia. Neuropsychologia. 2002;40(6):655-65.

18 Kessels RP, Gerritsen L, Montagne B, Ackl N, Diehl J, Danek A. Recognition of facial expressions of different emotional intensities in patients with frontotemporal lobar degeneration. Behav Neurol. 2007;18(1):31-6.

19 Werner KH, Roberts NA, Rosen HJ, Dean DL, Kramer JH, Weiner MW, et al. Emotional reactivity and emotion recognition in frontotemporal lobar degeneration. Neurology. 2007 Jul;69(2):148-55.

20 Kumfor F, Piguet 0. Disturbance of emotion processing in frontotemporal dementia: a synthesis of cognitive and neuroimaging findings. Neuropsychol Rev. 2012 Sep;22(3):280-97.

21 Goodkind MS, Sturm VE, Ascher EA, Shdo SM, Miller BL, Rankin KP, et al. Emotion recognition in frontotemporal dementia and Alzheimer's disease: A new film-based assessment. Emotion. 2015 Aug;15(4):416-27.

22 Rosen HJ, Perry RJ, Murphy J, Kramer JH, Mychack P, Schuff N, et al. Emotion comprehension in the temporal variant of frontotemporal dementia. Brain. 2002 Oct;125(Pt 10):2286-95.

23 Rosen HJ, Pace-Savitsky K, Perry RJ, Kramer JH, Miller BL, Levenson RW. Recognition of emotion in the frontal and temporal variants of frontotemporal dementia. Dement Geriatr Cogn Disord. 2004;17(4):277-81.

24 Kumfor F, Miller L, Lah S, Hsieh S, Savage S, Hodges JR, et al. Are you really angry? The effect of intensity on facial emotion recognition in frontotemporal dementia. Soc Neurosci. 2011;6(5-6):502-14.

25 Couto B, Manes F, Montañés P, Matallana D, Reyes P, Velasquez M, et al. Structural neuroimaging of social cognition in progressive non-fluent aphasia and behavioral variant of frontotemporal dementia. Front Hum Neurosci. 2013 Aug; 7:467.

26 Marshall CR, Hardy CJ, Russell LL, Bond RL, Sivasathiaseelan H, Greaves C, et al. The functional neuroanatomy of emotion processing in frontotemporal dementias. Brain. 2019 Sep;142(9):2873-87.

27 Cavallo M, Adenzato M, Macpherson SE, Karwig G, Enrici I, Abrahams S. Evidence of social understanding impairment in patients with amyotrophic lateral sclerosis. PLoS One. 2011;6(10):e25948. 
28 Savage SA, Lillo P, Kumfor F, Kiernan MC, Piguet O, Hodges JR. Emotion processing deficits distinguish pure amyotrophic lateral sclerosis from frontotemporal dementia. Amyotroph Lateral Scler Frontotemporal Degener. 2014 Mar;15(1-2):39-46.

29 Ghosh BC, Rowe JB, Calder AJ, Hodges JR, Bak TH. Emotion recognition in progressive supranuclear palsy. Neurol Neurosurg Psychiatry. 2009 Oct;80(10):1143-5.

30 Neary D, Snowden JS, Gustafson L, Passant U, Stuss D, Black S, et al. Frontotemporal lobar degeneration: a consensus on clinical diagnostic criteria. Neurology. 1998 Dec;51(6):1546-54.

31 Strong MJ, Abrahams S, Goldstein LH, Woolley S, Mclaughlin P, Snowden J, et al. Amyotrophic lateral sclerosis - frontotemporal spectrum disorder (ALS-FTSD): revised diagnostic criteria. Amyotroph Lateral Scler Frontotemporal Degener. 2017 May;18(3-4):153-74.

32 Mekala S, Paplikar A, Mioshi E, Kaul S, Divyaraj G, Coughlan G, et al. Dementia diagnosis in seven languages: the Addenbrooke's Cognitive Examination-III in India. Arch Clin Neuropsychol. 2020 Jul;35(5):528-38.

33 Grace J, Malloy P. FrSBe, frontal systems behavior scale: Professional manual. Psychological Assessment Resources; 2001.

34 Shobini L. Rao, Subbakrishna DK, Gopukumar K. NIMHANS Neuropsychology Battery- Manual. Bangalore, India: National Institute of Mental Health and Neurosciences; 2004.

35 Iyer GK, Paplikar A, Alladi S, Dutt A, Sharma M, Mekala S, et al. Standardising Dementia Diagnosis Across Linguistic and Educational Diversity: Study Design of the Indian Council of Medical Research-Neurocognitive Tool Box (ICMR-NCTB). J Int Neuropsychol Soc. 2020 Feb;26(2):172-86.

36 Mekala S. Social cognition and semantic memory in the cultural context of Indian healthy and neurologically diseased population [Doctoral Thesis]. Hyderabad (India) Nizam's Institute of Medical Sciences; 2013 [unpublished data].

37 Elamin M, Pender N, Hardiman O, Abrahams S. Social cognition in neurodegenerative disorders: a systematic review. J Neurol Neurosurg Psychiatry. 2012 Nov;83(11):1071-9.

38 Gorno-Tempini ML, Dronkers NF, Rankin KP, Ogar JM, Phengrasamy L, Rosen HJ, et al. Cognition and anatomy in three variants of primary progressive aphasia. Ann Neurol. 2004 Mar;55(3):335-46.

39 Hodges JR, Patterson K. Nonfluent progressive aphasia and semantic dementia: a comparative neuropsychological study. J Int Neuropsychol Soc. 1996 Nov;2(6):511-24.

40 Huey ED, Goveia EN, Paviol S, Pardini M, Krueger F, Zamboni G, et al. Executive dysfunction in frontotemporal dementia and corticobasal syndrome. Neurology. 2009; 3;72(5):453-9.

41 Roca M, Manes F, Gleichgerrcht E, Watson P, Ibáñez A, Thompson R, et al. Intelligence and executive functions in frontotemporal dementia. Neuropsychologia. 2013 Mar;51(4):725-30.

42 Kobylecki C, Jones M, Thompson JC, Richardson AM, Neary D, Mann DM, et al. Cognitive-behavioural features of progressive supranuclear palsy syndrome overlap with frontotemporal dementia. J Neurol. 2015;262(4): 916-22.

43 Kasper E, Schuster C, Machts J, Bittner D, Vielhaber S, Benecke R, et al. Dysexecutive functioning in ALS patients and its clinical implications. Amyotroph Lateral Scler Frontotemporal Degener. 2015 Jun;16(3-4):160-71.

44 Stojkovic T, Stefanova E, Pekmezovic T, Peric S, Stevic Z. Executive dysfunction and survival in patients with amyotrophic lateral sclerosis: preliminary report from a Serbian centre for motor neuron disease. Amyotroph Lateral Scler Frontotemporal Degener. 2016 Oct - Nov; 17(7-8):543-7.

45 Rusina R, Kovacs GG, Fiala J, Hort J, Ridzoň P, Holmerová I, et al. FTLD-TDP with motor neuron disease, visuospatial impairment and a progressive supranuclear palsy-like syndrome: broadening the clinical phenotype of TDP-43 proteinopathies. A report of three cases. BMC Neurol. 2011 May;11(1):50.

46 Marczinski CA, Davidson W, Kertesz A. A longitudinal study of behavior in frontotemporal dementia and primary progressive aphasia. Cogn Behav Neurol. 2004 Dec;17(4):185-90.

47 Harciarek M, Jodzio K. Neuropsychological differences between frontotemporal dementia and Alzheimer's disease: a review. Neuropsychol Rev. 2005 Sep;15(3):131-45.

48 Peters F, Perani D, Herholz K, Holthoff V, Beuthien-Baumann B, Sorbi S, et al. Orbitofrontal dysfunction related to both apathy and disinhibition in frontotemporal dementia. Dement Geriatr Cogn Disord. 2006;21(5-6): 373-9.

49 Zamboni G, Huey ED, Krueger F, Nichelli PF, Grafman J. Apathy and disinhibition in frontotemporal dementia: insights into their neural correlates. Neurology. 2008 Sep;71(10):736-42.

50 Go C, Mioshi E, Yew B, Hodges JR, Hornberger M. Neural correlates of behavioural symptoms in behavioural variant frontotemporal dementia and Alzheimer's disease: employment of a visual MRI rating scale. Dement Neuropsychol. 2012 Jan-Mar;6(1):12-7.

51 Tippett DC, Thompson CB, Demsky C, Sebastian R, Wright A, Hillis AE. Differentiating between subtypes of primary progressive aphasia and mild cognitive impairment on a modified version of the Frontal Behavioral Inventory. PLoS One. 2017 Aug;12(8):e0183212.

52 Rosen HJ, Allison SC, Schauer GF, Gorno-Tempini ML, Weiner MW, Miller BL. Neuroanatomical correlates of behavioural disorders in dementia. Brain. 2005 Nov;128(Pt 11):2612-25.

53 Dermody N, Wong S, Ahmed R, Piguet O, Hodges JR, Irish M. Uncovering the neural bases of cognitive and affective empathy deficits in Alzheimer's disease and the behavioral-variant of frontotemporal dementia. J Alzheimers Dis. 2016 May;53(3):801-16. 
54 Eslinger PJ, Moore P, Anderson C, Grossman M. Social cognition, executive functioning, and neuroimaging correlates of empathic deficits in frontotemporal dementia. J Neuropsychiatry Clin Neurosci. 2011;23(1): 74-82.

55 Rankin KP, Gorno-Tempini ML, Allison SC, Stanley CM, Glenn S, Weiner MW, et al. Structural anatomy of empathy in neurodegenerative disease. Brain. 2006 Nov;129(Pt 11):2945-56.

56 Hazelton J, Irish M, Hodges J, Piguet O, Kumfor F. Cognitive and Affective Empathy Disruption in Non-Fluent Primary Progressive Aphasia syndromes. Brain Impair. 2017;18(1):117-29.

57 Irish M, Hodges JR, Piguet O. Right anterior temporal lobe dysfunction underlies theory of mind impairments in semantic dementia. Brain. 2014 Apr;137(Pt 4):1241-53.

58 Shdo SM, Ranasinghe KG, Gola KA, Mielke CJ, Sukhanov PV, Miller BL, et al. Deconstructing empathy: neuroanatomical dissociations between affect sharing and prosocial motivation using a patient lesion model. Neuropsychologia. 2018 Jul 31;116(Pt A):126-35.

59 Cerami C, Dodich A, Canessa N, Crespi C, Iannaccone S, Corbo M, et al. Emotional empathy in amyotrophic lateral sclerosis: a behavioural and voxel-based morphometry study. Amyotroph Lateral Scler Frontotemporal Degener. 2014 Mar;15(1-2):21-9.

60 Mueller K, Jech R, Bonnet C, Tintěra J, Hanuška J, Möller HE, et al.; FTLDc Study Group. Disease-specific regions outperform whole-brain approaches in identifying progressive supranuclear palsy: a Multicentric MRI Study. Front Neurosci. 2017 Mar;11:100.

61 Fernandez-Duque D, Black SE. Impaired recognition of negative facial emotions in patients with frontotemporal dementia. Neuropsychologia. 2005;43(11):1673-87.

62 Kipps CM, Nestor PJ, Acosta-Cabronero J, Arnold R, Hodges JR. Understanding social dysfunction in the behavioural variant of frontotemporal dementia: the role of emotion and sarcasm processing. Brain. 2009b Mar; 132(Pt 3):592-603. 